\title{
Symbolic Algebra Programming for Analyzing the Long Run Dynamics of Economic Models: An Investigation of Overlapping Generations Models
}

\author{
Gary S. Anderson \\ Department of Economics \\ University of Maryland \\ College Park, MD ${ }^{1}$
}

March 31, 1992

Revised January 6, 1998

\footnotetext{
${ }^{1}$ National Science Foundation Grant SES-9012166 supported this work at the University of Maryland, College Park, MD. I prepared this paper for presentation at the Conference on Computing for the Social Sciences, May 4-7, 1992, University of Michigan, Ann Arbor.
} 


\begin{abstract}
Economists have long used overlapping generations models to explore important empirical and theoretical issues in public finance, development, international trade, savings and monetary policy. Recently, some researchers have criticized the way these and other models characterize the long run tendency of the economy. If the equations which codify the assumptions in the models can display bizarre behavior, the models could give misleading forecasts of the behavior of the economy. By studying the mathematical equations which economists use to codify and apply these models, I am investigating the relationship between the empirically determined parameters and the corresponding long run properties of the models.

This paper shows how symbolic algebra programs can facilitate the analysis of the dynamics of these non-linear equation systems. I have used the symbolic algebra capabilities of Mathematica to develop a collection of programs for analyzing the asymptotic behavior of economic models. These symbolic programming algorithms implement a set of algorithms orignally designed for numerical processing. The paper shows how to use these tools to derive formulae for characterizing the long run dynamics of overlapping generations models. The powerful symbolic and algebraic manipulation tools make it possible to analytically explore the subtle transitions between generic classes of long run behavior for these models. The paper develops formulae for characterizing the asymptotic behavior of the model for plausible ranges of the parameters. These results provide insights about features of these models which are useful for both theoretical and empirical economists.
\end{abstract}




\section{Introduction}

Nonlinear dynamic models have served as important tools for much of modern macroeconomics[. blanchard fischer, sargeant dynamic .]. The Ramsey neoclassical growth model, and overlapping generation models are but two examples of mainstream macroeconomic models which posit a nonlinear specification of the relationship between economic variables over time. Economists use these models in the class room, in theoretical and empirical analysis of important aggregate relationships, and for investigating the potential impact of monetary, and fiscal policy. The continued increase in computing speed and declining cost of computing for economists probably means nonlinear models will become even more important for future econometric and policy analysis.

Some of these nonlinear models come from dynamic optimization of the choice problem facing individual economic agents or a central planner. Economists often use first order conditions from dynamic optimization problems and a simplifying certainty equivalence assumption to characterize the behavior of economic agents. This approach leads to models which possess the "saddle point property." Simulation and estimation of these models involves solving a nonlinear two point boundary value problem with one endpoint arbitrarily far off in the future.

Although dynamic nonlinear models can have very complicated behavior[. guckenheimer holmes .] , economists who use these models often implicitly assume that these mainstrem economic models have a fixed point to which model solutions converge. Unfortunately, it is difficult to guarantee that a given nonlinear specification will have the appropriate asymptotic properties for all plausible values of model parameters. It is becoming clear that it is important to be aware of and accommodate a more comprehensive set of possible asymptotic solutions when applying these models or while searching parameter space when estimating the coefficients in such models.

\subsection{Complicated Dynamics are Potentially Present in Tra- ditional Economic Models}

Recently, some economists have begun to embrace the potentially complex nonlinear asymptotic behavior of nonlinear dynamic economic models as an important topic of investigation. Gale first pointed out the existence of equilibrium cycles in overlapping generations models[. gale .]. Benhabib and Rustichini[. benhabib rustichini .] describe a neoclassical growth model with equilibrium limit cycles with the idea of convincing his colleagues that this is not unusual for dynamic nonlinear models. Jullien[. jullien .] investigates bifurcations of solutions of a nonlinear model to establish the existence of cycles. Jullien demonstrates the equivalence of an overlapping generations model and a one dimensional system to prove that equilibrium cycles and chaotic solutions are possible.

Grandmont[. grandmont .] studies the existence of sunspot equilibria in nonlinear models by investigating their potentially complex asymptotic dynamics. 
Laitner describes how complicated dynamics is a prerequisite for the existence of sunspot equilibria[. laitner sunspot .]. James Peck performs similar analysis on overlapping generations models to identify situations where there are sunspot equilibria[. peck .].

The possible existence of complicated long run dynamics has several potentially important implications for the use of nonlinear models in economics.

\subsubsection{Implications for Econometrics}

The existing methodologies for simulating and estimating these models often encounter problems which are hard to diagnose. During the model development process economists conceive models with appropriate asymptotic properties, but during estimation, hill climbing routines control the parameters and the asymptotic properties. A fixed point with the appropriate stability property may evolve into a periodic point, or disappear or loose the saddle point property frustrating the function evaluation phase of the estimation process.

A robust method for simulating and estimating these models must carefully investigate the asymptotic behavior of the models in order to identify problems caused by a failure of the solution methodology, poor model specification, bad parameter selection, or programmer error.

\subsubsection{Implications for Our Basic Understanding of a Competitive System}

Analysis of the overlapping generations model and neoclassical growth models suggests that a competitive system may have an endogenous business cycle. Benhabib and Rustichini[. benhabib rustichini .] describe a neoclassical growth model with equilibrium limit cycles. They show that the perfect competition assumption does not rule out persistent endogenous cycles.

There is a close relationship between the existence of multiple solutions, indeterminacy of perfect foresight paths and bifurcations of fixed points on the one hand and the existence of sunspot equilibria on the other[. muller woodford, peck, laitner sunspots .]. When there are sun spot equilibria, self fulfilling expectations are often insufficient to uniquely determine the future values of economic variables. In this case, it may be impossible for individual agents to learn the underlying fundamental parameters of the model by observing economic timeseries. In such a world, "animal spirits" can play an important role in conditioning economic agents' expectations of the evolution of economic variables.

\subsection{Tools and Methodology From Dynamic Systems Anal- ysis Useful in Diagnosing or Exploiting Complicated Dynamic Behavior of Nonlinear Economic Systems}

When nonlinear models possess fixed points or limit cycles, a linear algebraic analysis of asymptotic properties can often determine the local existence and 
uniqueness of convergent paths. The local analysis of a nonlinear dynamical system should begin with a study of the first order or linear behavior of the equation system[. devaney, guckenheimer holmes .]. ${ }^{1}$

For "saddle point" models, linear algebraic analysis of the asymptotic behavior provides linear restrictions on the solution paths that are useful for computing nonlinear solutions. Economically meaningful solutions require appropriate asymptotic convergence properties. Laitner[. laitner definition .] provides a definition of stability for nonlinear perfect foresight models. Anderson-Moore[. anderson linear algebraic .] describe a technique for checking the appropriateness of the asymptotic properties of linear models. The Anderson-Moore technique is easily extended to nonlinear systems, and Mathematica, a symbolic algebra program, provides powerful tools for automating the symbolic computations involved in applying these asymptotic stability checks.

\section{Nonlinear Dynamic Economic Model Specifi- cation}

\subsection{General Formulation}

Consider the model

$$
\begin{gathered}
h\left(x_{t-\tau}, x_{t-\tau+1}, \ldots, x_{t+\theta-1}, x_{t+\theta}\right)=0 \\
t=0, \ldots, \infty
\end{gathered}
$$

where $x \in \Re^{L}$ and $h: \Re^{L(\tau+1+\theta)} \rightarrow \Re^{L}$. We want to determine the solutions to equation system 1 with initial conditions

$$
x_{i}=\bar{x}_{i} \text { for } i=-\tau, \ldots,-1
$$

satisfying

1. A fixed point

$$
\lim _{t \rightarrow \infty} x_{t}=x^{*}
$$

or

\section{A Limit Cycle}

$$
\lim _{k \rightarrow \infty}\left[\begin{array}{c}
x_{p k} \\
x_{p k+1} \\
\vdots \\
x_{p k+(p-1)}
\end{array}\right]=\left[\begin{array}{c}
x_{0}^{*} \\
x_{1}^{*} \\
\vdots \\
x_{p-1}^{*}
\end{array}\right]
$$

Where $\mathrm{p}$ is the periodicity of the limit cycle.

The literature contains many models which are special cases of equation system 1.

\footnotetext{
${ }^{1}$ Terms higher than first order must be considered at nonhyperbolic periodic points.
} 


\subsection{Several Example Dynamic Nonlinear Economic Mod- els}

\subsubsection{The Overlapping Generations Model of Benhabib and Laroque}

Benhabib and Laroque[. benhabib laroque .] present an overlapping generations model with money, labor, and a good which workers can consume or store as capital. Benhabib and Laroque demonstrate that there is a one to one correspondence between competitive equilibria of this model and solutions to the three equation system

$$
\begin{gathered}
f_{k}\left(k_{t}, l_{t+1}\right) f_{l}\left(k_{t-1}, l_{t}\right) U_{1}^{\prime}\left(f_{k}\left(k_{t}, l_{t+1}\right) f_{k}\left(k_{t-1}, l_{t}\right) l_{t}\right)- \\
U_{2}^{\prime}\left(l_{t}\right)=0 \\
k_{t}+M q_{t}-f_{l}\left(k_{t-1}, l_{t}\right)=0 \\
q_{t+1}-f_{k}\left(k_{t}, l_{t+1}\right) q_{t}=0
\end{gathered}
$$

Benhabib and Laroque use this model to demonstrate that cyclical equilibria exist, and to develop an interpretable parameterization of the model. They study the behavior of their model near the golden rule equilibria and apply results from bifurcation theory to show the existence of limit cycles.

\subsubsection{The Overlapping Generations Model of Jullien}

Jullien[. jullien .] also studies an overlapping generations model with production where $m_{t}$ represents real money balances, $k_{t}$ represents capital, $W\left(k_{t}\right)$ is a wage function reflecting the marginal product of labor from a constant returns production function, and $S\left(W\left(k_{t}\right), f^{\prime}\left(k_{t}\right)\right)$ is a savings function obtained from intertemporal utility maximization. His system is also of the form of equation system 1.

$$
\begin{gathered}
m_{t}+k_{t}=S\left(W\left(k_{t-1}\right), f^{\prime}\left(k_{t}\right)\right) \\
m_{t+1}=f^{\prime}\left(k_{t}\right) m_{t}
\end{gathered}
$$

He demonstrates the possibility of cyclical and chaotic behavior by recasting the model as a one dimensional system and using well known results characterizing the global properties of one dimensional maps.

\subsubsection{A Certainty Equivalence Formulation of a Real Business Cycle Model}

McCallum[. barro business cycle.] describes a real business cycle model:

$$
\begin{gathered}
c_{t}+k_{t}=z_{t} f\left(n_{t}, k_{t-1}\right)+(1-\delta) k_{t-1} \\
u_{1}\left(c_{t}, 1-n_{t}\right)-\lambda_{t}=0 \\
u_{2}\left(c_{t}, 1-n_{t}\right)=\lambda_{t} z_{t} f_{1}\left(n_{t}, k_{t-1}\right) \\
\lambda_{t}=\beta \lambda_{t+1}\left[z_{t+1} f_{2}\left(n_{t+1}, k_{t}\right)+1-\delta\right] \\
\left(z_{t}-z^{*}\right)=\rho\left(z_{t-1}-z^{*}\right)
\end{gathered}
$$

This model is also of the form of equation system 1 . 


\subsubsection{A Money Demand Model}

Consider the three equation nonlinear system

$$
\begin{gathered}
\ln \frac{m_{t}}{p_{t}}=\alpha+\beta \ln \left(\rho+\left(\frac{p_{t+1}-p_{t}}{p_{t}}\right)\right) \\
m_{t}-m_{t-1}=\gamma\left(m_{t-1}-\mu\right)+\delta s_{t} \\
s_{t}=\lambda s_{t-1}\left(1-s_{t-1}\right)
\end{gathered}
$$

Where $\tau=1, \theta=1$, and $0 \leq \lambda, \alpha<0, \beta<0, \rho>0, \gamma<0$, and $m^{*}>0$ exogenously given.

This example augments a simple forward looking money demand model with a quadratic map. The quadratic map is a much studied nonlinear function whose asymptotic properties are well known[. devaney .]. As we vary the parameter $\lambda$ we can study a model with fixed points, limit cycles, and with more complicated invariant sets.

There are several widely know techniques for computing solutions to equation system 1 when $h$ is linear[. blanchard kahn .]. This paper adopts the method of Anderson-Moore[. anderson linear algebraic .] to determine the existence, and local uniqueness of the solutions to equation system 1.

\section{A General Methodology for Analyzing the Asymptotic Properties of Nonlinear Dynamic Economic Models}

\subsection{The Anderson-Moore Linear Algebraic Technique for Solving Linear Perfect Foresight Models}

Anderson-Moore[. anderson linear algebraic .] present a fail-safe method for analyzing any linear perfect foresight model. They describe a procedure which either computes the reduced form solution or indicates why the model has no reduced form.

Anderson-Moore[. anderson linear algebraic .] outline a procedure that computes solutions for structural models of the form

$$
\sum_{i=-\tau}^{\theta} H_{i} X_{t+i}=0, t \geq 0
$$

with initial conditions

$$
X_{i}=x_{i}, i=-\tau, \ldots,-1
$$

where both $\tau$ and $\theta$ are non-negative, and $X_{t}$ is an $\mathrm{L}$ dimensional real vector.

Anderson-Moore[. anderson efficient .] provides a constructive proof of the following theorem. 
Theorem 1 If the $L$ by $L$ real coefficient matrices $H_{i}: i=-\tau, \ldots, \theta$ satisfy the following two restrictions:

1. The origin is the unique steady state of equation 3. That is, if

$$
\left(\sum_{i=-\tau}^{\theta} H_{i}\right) X^{*}=0, \Rightarrow X^{*}=0
$$

2. Corresponding to any initial conditions $X_{i}=x_{i}: i=-\tau, \ldots,-1$, equation 3 has a unique solution $X_{t}: t \geq 0$ such that

$$
\lim _{t \rightarrow \infty} X_{t}=0
$$

then the model has a reduced-form representation

$$
X_{t}=\sum_{i=-\tau}^{-1} B_{i} X_{t+i}, t>0
$$

generating the unique solution $X_{t}: t \geq 0$ such that

$$
\lim _{t \rightarrow \infty} X_{t}=0 .
$$

Anderson-Moore[. anderson linear algebraic .] presents a constructive procedure for analyzing linear perfect foresight models. Given the coefficient matrix

$$
\left[\begin{array}{lll}
H_{-\tau} & \ldots & H_{\theta}
\end{array}\right]
$$

the procedure computes the reduced form coefficient matrix

$$
\left[\begin{array}{lll}
B_{-\tau} & \ldots & B_{-1}
\end{array}\right]
$$

for any model satisfying assumptions 1 and 2. If the model does not satisfy assumptions 1 and 2, the procedure indicates whether there are no convergent solutions or a multiplicity of convergent solutions.

In order to compute the matrix $\mathrm{B}$ the algorithm first computes a matrix $\mathrm{Q}$ which embodies constraints which come from computing a state space transition matrix and linear constraints that guarantee asymptotic convergence to the saddle point. The algorithm described in section 3.2 uses this matrix Q.

\subsubsection{Summary of the Procedure}

This section summarizes the procedure presented in Anderson Moore[. anderson linear algebraic .] 


\section{Initialization}

1. Verify that $\left(\sum_{i=-\tau}^{\theta} H_{i}\right)$ is full rank. If it is singular, the steady state is not unique; stop.

2. If it is non-singular, initialize

$$
\begin{aligned}
H^{(0)} & :=\left[\begin{array}{lll}
H_{-\tau} & \ldots & H_{\theta}
\end{array}\right] \\
Q^{(0)} & :=\text { nullmatrix }
\end{aligned}
$$

\section{Auxiliary Initial Conditions}

1. Compute the singular values, $\left\{\mu_{i}: i=1, \ldots, L\right\}$, and singular vectors, $\mathrm{V}$, of $H_{\theta}^{\left(k^{*}\right)}$. Sort the $\mu_{i}$ small-to-large and order the columns of $\mathrm{V}$ conformably. If $\mu_{i} \neq 0, i=1, \ldots, L$, then $H_{\theta}^{\left(k^{*}\right)}$ is non-singular; go to step 1 of the paragraph on stability conditions below.

2. $H_{\theta}^{\left(k^{*}\right)}$ is singular. Premultiply the coefficient matrix by $V^{T}$ to annihilate L-rank $\left(H_{\theta}^{\left(k^{*}\right)}\right)$ rows of $H_{\theta}^{\left(k^{*}\right)}$.

$$
\tilde{H}^{(k)}:=V^{T} H^{(k)}
$$

3. Partition the coefficient matrix as

\begin{tabular}{|c|c|}
\hline $\mathrm{q}$ & 0 \\
\hline $\mathrm{r}$ & $:=\tilde{H}^{(k)}$ \\
\hline
\end{tabular}

The matrix q has $\mathrm{L}-\operatorname{rank}\left(H_{\theta}^{\left(k^{*}\right)}\right)$ rows and $\mathrm{L}(\tau+\theta)$ columns; $\mathrm{r}$ has $\operatorname{rank}\left(H_{\theta}^{(k)}\right)$ rows and $\mathrm{L}(\tau+1+\theta)$ columns.

4. Include $\mathrm{q}$ among the auxiliary initial conditions

$$
Q^{(k+1)}:=\begin{array}{|c|}
Q^{(k)} \\
\hline \mathrm{q} \\
\hline
\end{array}
$$

Now, shift the sub-matrix q L columns to the right in $\mathrm{H}$,

$H^{(k+1)}:=$\begin{tabular}{|c|c|}
\hline 0 & $\mathrm{q}$ \\
\hline \multicolumn{3}{|c|}{$\mathrm{r}$} \\
\hline
\end{tabular}

Repeat these four steps until $H_{\theta}^{(k)}$ is non-singular. Let $k^{*}$ denote this final value of $\mathrm{k}$ 


\section{Stability Conditions}

1. $H_{\theta}^{\left(k^{*}\right)}$ is non-singular. Solve for coefficients expressing $X_{t+\theta}$ in terms of $X_{t-\tau} \ldots X_{t+\theta-1}$.

$$
\Gamma:=-\left(H_{\theta}^{\left(k^{*}\right)}\right)^{-1}\left[\begin{array}{lll}
H_{-1}^{\left(k^{*}\right)} & \ldots H_{(\theta-1)}^{\left(k^{*}\right)}
\end{array}\right]
$$

2. Construct the first order state space transition matrix

$$
A:=\left[\begin{array}{cc}
0 & I \\
\Gamma
\end{array}\right]
$$

3. Compute $\mathrm{W}$, a matrix of row vectors spanning the left invariant subspace of A associated with roots outside the open unit disk. One can use the routine HQR3 presented by G. Stewart[. stewart hqr3 .]. The matrix W contains the stability conditions which guarantee the saddle point property[. anderson efficient .].

\section{Reduced Form}

1. Concatenate the auxiliary initial conditions with the stability conditions.

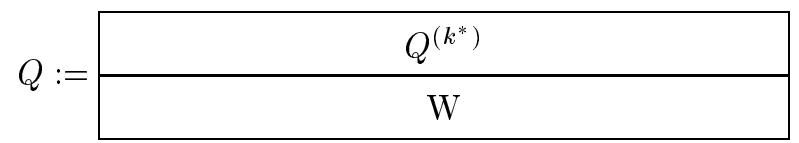

\section{Partition Q.}

$$
\left[\begin{array}{ll}
Q_{L} & Q_{R}
\end{array}\right]:=Q
$$

where $Q_{L}$ has $L \tau$ columns and $Q_{R}$ has $L \theta$ columns.

3. Let $\mathrm{n}$ be the number of rows in $\mathrm{Q}$.

(a) If $n<L \theta$, then assumption 2 is violated; there are many solutions converging to the origin for any initial condition. Stop.

(b) If $n>L \theta$ or $n=L \theta$ and $Q_{R}$ is singular, then assumption 2 is violated; there exist initial conditions for which there are no solutions converging to the steady state. Stop.

(c) If $n=L \theta$ and $Q_{R}$ is non-singular, then set

$$
\begin{aligned}
& {\left[\begin{array}{lll}
B_{-\tau} & \ldots & B_{-1}
\end{array}\right]:=\text { the first } \mathrm{L} \text { rows of }-Q_{R}^{-1} Q_{L} \text { and }} \\
& X_{t}=\sum_{i=-\tau}^{-1} B_{i} X_{t+i}, t \geq 0
\end{aligned}
$$

is the unique solution converging to the steady state for any initial conditions. Stop.

End. 


\subsection{A Nonlinear Extension of the Anderson-Moore Tech- nique}

An investigation of the asymptotic dynamics of nonlinear difference equations can usefully begin with an analysis of the asymptotically linear behavior near fixed points and limit cycles[. guckenheimer holmes, laitner definition, laitner steady states econometrica .].

Since $h$ is nonlinear, we will compute approximate solutions to equation system 1 by linearizing the nonlinear $h$ constraints in equation system 1 at the fixed point or limit cycle.

\subsubsection{Fixed Points}

Compute the steady state value $x^{*}$ satisfying

$$
h\left(x^{*}, \ldots, x^{*}\right)=0
$$

Near the steady state, the linear first-order Taylor expansion of $\mathrm{h}$ about $x^{*}$ provides a good approximation to the function $h$.

$$
\left.h\left(x_{t-\tau}, \ldots, x_{t+\theta}\right) \approx \sum_{i=-\tau}^{\theta} H_{i}\right|_{x^{*}}\left(x_{t+i}-x^{*}\right)
$$

We can apply the techniques presented in[. anderson linear algebraic .] to determine the existence and uniqueness of perfect foresight solutions near the steady state.

That stability analysis will produce a matrix $Q$ which restricts values of the endogenous variables to the stable subspace of the linearized system. For trajectories which approach a steady state, we can ultimately replace the nonlinear system with the constraints codified in the matrix $Q$.

$$
Q\left[\begin{array}{c}
x_{T-\tau}-x^{*} \\
\vdots \\
x_{T}-x^{*} \\
\vdots \\
x_{T+\theta}-x^{*}
\end{array}\right]=0
$$

\subsubsection{Limit Cycles}

Compute the periodic points $x_{1}^{*}, x_{2}^{*}, \ldots, x_{p}^{*}$ satisfying

$$
\left[\begin{array}{c}
h\left(x_{-\tau}^{*}, \ldots, x_{1}^{*}, \ldots, x_{\theta}^{*}\right) \\
h\left(x_{1-\tau}^{*}, \ldots, x_{2}^{*}, \ldots, x_{1+\theta}^{*}\right) \\
\vdots \\
h\left(x_{p-\tau}^{*}, \ldots, x_{p}^{*}, \ldots, x_{p+\theta}^{*}\right)
\end{array}\right]=0
$$


Near a limit cycle, the linear first-order Taylor expansion of $h$ about $x_{t}^{*}$ provides a good approximation to the function $h$.

$$
\left.h\left(x_{t-\tau}, \ldots, x_{t+\theta}\right) \approx \sum_{i=-\tau}^{\theta} H_{i}\right|_{x_{i-\tau}^{*} \ldots x_{i+\theta}^{*}}\left(x_{t+i}-x_{i}^{*}\right)
$$

Define $(\bar{\theta}+1)$ matrices of dimension $p L \times p L$

$$
\begin{aligned}
& \bar{H}_{0}=\left[\begin{array}{ccc}
\left.H_{-\tau}\right|_{x_{1-\tau}^{*} \ldots x_{1+\theta}^{*}} & \ldots & \left.H_{p-\tau}\right|_{x_{1-\tau}^{*} \ldots x_{1+\theta}^{*}} \\
& \ddots & \vdots \\
& & \left.H_{-\tau}\right|_{x_{p-\tau}^{*} \ldots x_{p+\theta}^{*}}
\end{array}\right] \\
& \bar{H}_{1}=\left[\begin{array}{ccc}
\left.H_{p-\tau+1}\right|_{x_{1-\tau}^{*} \ldots x_{1+\theta}^{*}} & \ldots & \left.H_{p-\tau+p}\right|_{x_{1-\tau}^{*} \ldots x_{1+\theta}^{*}} \\
\vdots & \vdots & \vdots \\
\left.H_{-\tau+1}\right|_{x_{p-\tau}^{*} \ldots x_{p+\theta}^{*}} & \ldots & \left.H_{p-\tau+1}\right|_{x_{p-\tau}^{*} \ldots x_{p+\theta}^{*}}
\end{array}\right] \\
& \bar{H}_{\bar{\theta}}=\left[\begin{array}{ccc}
\left.H_{-\theta}\right|_{x_{1-\tau}^{*} \ldots x_{1+\theta}^{*}} & & \\
\vdots & \ddots & \\
\left.H_{\theta-p}\right|_{x_{p-\tau}^{*} \ldots x_{p+\theta}^{*}} & \cdots & \left.H_{\theta}\right|_{x_{p-\tau}^{*} \ldots x_{p+\theta}^{*}}
\end{array}\right]
\end{aligned}
$$

where $\bar{\theta}$ is the smallest integer greater than $(\tau+\theta+p) / p$. The $\bar{H}_{0}, \ldots, \bar{H}_{\bar{\theta}}$ make up a linear system of the form explored in[. anderson linear algebraic .].

Analysis of this system will produce a matrix of constraints, $Q$, which guarantee that the trajectories near the limit cycle converge to the limit cycle. For trajectories of the original system which converge to this limit cycle, we can ultimately replace the nonlinear system of equations with the matrix of constraints, $Q$.

$$
Q\left[\begin{array}{c}
x_{-\tau}-x_{-\tau}^{*} \\
\vdots \\
x_{0}-x_{0}^{*} \\
\vdots \\
x_{p \theta}-x_{p \theta}^{*}
\end{array}\right]=0
$$

Although the algorithm and all the models presented here are discrete time models, the technique is easily modified to accommodate continuous time models.

The asymptotic linearization of a nonlinear system provides a succinct characterization of evolution of trajectories so long as the magnitudes of the eigenvalues are not equal to one. The asymptotic behavior of nonlinear models at so called non hyperbolic points is more subtle[. guckenheimer holmes .]. Methods 
for characterizing the asymptotic behavior still rely on identifying hyperbolic points by linear algebraic means and renormalizing the system to analyze the behavior of higher order terms in the subspace associated with the unit modulus eigenvectors. Nonhyperbolic points are important in dynamic economic models because, as Laitner[. laitner steady perfect foresight .] and Peck[. peck .] have noted, there is a close association between nonhyperbolicity and the existence of sunspot equilibria. In addition, Benhabib and Laroque[. benhabib jafarey .], Reichlin[. reichlin .] have emphasized the role of nonhyperbolicity in constructing equilibrium cycles at the critical bifurcation values of model parameters.

\subsection{Symbolic Processing Routines}

This section describes the several symbolic processing routines developed to carry out the analysis of non-linear economic models. ${ }^{2}$

\subsection{1 derivativesOfVectorFunction}

This routine takes a list of functions and a list of variables as inputs and returns a matrix of derivatives.

$$
\text { derivativesOfVectorFunction }[f, x] \rightarrow \frac{\partial f}{\partial x}
$$

\subsection{2 makeSymbolicAsymptoticHMatrix}

This routine takes a non linear model specification, and a periodicity as input and generates a symbolic matrix representing the asymptotic $\bar{H}_{i}$ described in equations $4-6$.

$$
\text { makeSymbolicAsymptoticHMatrix }[(f, x, \alpha,(\tau, \theta)), p] \rightarrow\left[\bar{H}_{0} \ldots \bar{H}_{\bar{\theta}}\right]
$$

\subsection{3 stateSpaceTransitionMatrix}

This routine takes a matrix of coefficients corresponding to the $H_{i}$ matrices of equation 3 and returns the three matrix results of the auxiliary initial conditions phase of the Anderson Moore algorithm: the matrix $A$, the matrix $Q^{\left(k^{*}\right)}$, and the matrix $H^{\left(k^{*}\right)}$

$$
\text { stateSpaceTransitionMatrix }\left[\left[H_{-\tau} \ldots H_{\theta}\right]\right] \rightarrow\left(A, Q^{\left(k^{*}\right)}, H^{\left(k^{*}\right)}\right)
$$

\subsection{4 eliminateInessentialLags}

This routine identifies superfluous columns and rows in the transition matrix thereby reducing the dimension of the state vector. It takes a transition matrix and an indicator vector of the same dimension as input. The routine returns

\footnotetext{
${ }^{2}$ These routines are available via anonymous ftp from info.umd.edu. Alternatively, one can contact the author via e-mail at gary_s_anderson@umail.umd.edu to request copies of the programs.
} 
a reduced dimension transition matrix with the corresponding elements deleted from the indicator vector.

$$
\text { eliminatelnessentialLags }[(A, i)] \rightarrow(\bar{A}, \bar{i})
$$

\subsection{The Analytic Implementation of the Anderson-Moore Saddle Point Analysis Routine Applied to the Exam- ple Nonlinear Models}

The algorithm described above was originally designed for use in estimation of linear rational expectations models and for estimation of nonlinear certainty equivalence models. In that context, it is usually necessary and appropriate to use numerical linear algebra routines to compute the required matrices. However, here, Mathematica provides tools for automating symbolic computation. In the present context it has proven worthwhile to use this symbolic algebra program to compute the matrices analytically.

\subsubsection{The Money Demand Model}

Now

$$
\begin{gathered}
H_{-1}=\left[\begin{array}{ccc}
0 & 0 & 0 \\
-(1+\gamma) & 0 & 0 \\
0 & 0 & \lambda\left(2 s_{t-1}-1\right)
\end{array}\right] \\
H_{0}=\left[\begin{array}{ccc}
\frac{1}{m_{t}} & \frac{\beta p_{t+1}-\rho p_{t}-\left(p_{t+1}-p_{t}\right)}{p_{t}\left(\rho p_{t}+\left(p_{t+1}-p_{t}\right)\right)} & 0 \\
1 & 0 & -\delta \\
0 & 0 & 1
\end{array}\right] \\
H_{1}=\left[\begin{array}{ccc}
0 & \frac{-\beta}{\rho p_{t}+\left(p_{t+1}-p_{t}\right)} & 0 \\
0 & 0 & 0 \\
0 & 0 & 0
\end{array}\right]
\end{gathered}
$$

We want to investigate the model with initial conditions

$$
\begin{aligned}
m_{0} & =\bar{m}_{0} \\
p_{0} & =\bar{p}_{0} \\
s_{0} & =\bar{s}_{0}
\end{aligned}
$$

and terminal conditions

$$
\lim _{t \rightarrow \infty}\left[\begin{array}{c}
m_{t} \\
p_{t} \\
s_{t}
\end{array}\right]=\left[\begin{array}{c}
m^{*} \\
p^{*} \\
s^{*}
\end{array}\right]
$$


with $s^{*}=0, \frac{\lambda-1}{\lambda} m^{*}=\mu-\frac{-\delta s^{*}}{\gamma} p^{*}=m^{*} \exp ^{-(\alpha+\beta \ln (\rho))}$, so that when $|\delta|<1$ the fixed point,

$$
\begin{gathered}
\left.H_{-1}\right|_{x^{*}}=\left[\begin{array}{ccc}
0 & 0 & 0 \\
-(1+\gamma) & 0 & 0 \\
0 & 0 & \lambda\left(2 s^{*}-1\right)
\end{array}\right] \\
\left.H_{0}\right|_{x^{*}}=\left[\begin{array}{ccc}
\frac{1}{m^{*}} & \frac{\beta-\rho}{\rho p^{*}} & 0 \\
1 & 0 & -\delta \\
0 & 0 & 1
\end{array}\right] \\
\left.H_{1}\right|_{x^{*}}=\left[\begin{array}{ccc}
0 & \frac{-\beta}{\rho p^{*}} & 0 \\
0 & 0 & 0 \\
0 & 0 & 0
\end{array}\right]
\end{gathered}
$$

so that applying the methods of [. anderson linear algebraix .] near the fixed point, the state space transition matrix is

$$
A=\left[\begin{array}{ccc}
(1+\gamma) & 0 & \delta \lambda\left(2 s^{*}-1\right) \\
\frac{\rho / \beta}{m^{*} / p^{*}} & \frac{\beta-\rho}{\beta} & 0 \\
0 & 0 & -\lambda\left(2 s^{*}-1\right)
\end{array}\right]
$$

The Q matrix consists of two shifted equations and one unstable left eigenvector if $\lambda>1$

$$
Q=\left[\begin{array}{cccccc}
-(1+\gamma) & 0 & 0 & 1 & 0 & -\delta \\
0 & 0 & \lambda\left(2 s^{*}-1\right) & 0 & 0 & 1 \\
0 & 0 & 0 & \frac{-\rho / \beta}{m^{*} / p^{*}} & \left(\frac{(\beta-\rho)}{\beta}+(1+\gamma)\right) & \frac{-\delta \frac{\rho \beta \beta}{m^{*} / p^{*}}}{\left(\frac{\beta-\rho}{\beta}+\delta\right)}
\end{array}\right]
$$

So that

$$
B=\left[\begin{array}{ccc}
1+\gamma & 0 & \delta \lambda-2 \delta \lambda s^{*} \\
\frac{(1+\gamma) \rho p^{*}}{(\beta \gamma+\rho) m^{*}} & 0 & \frac{\delta \lambda \rho(-\beta+\rho) p^{*}\left(-1+2 s^{*}\right)}{(\beta \gamma+\rho) m^{*}\left(\beta-\beta \lambda-\rho+2 \beta \lambda s^{*}\right)} \\
0 & 0 & \lambda-2 \lambda s^{*}
\end{array}\right]
$$

In this simple example I have grafted a nonlinear equation with known asymptotic properties onto a simple model which typically has only fixed points in order to demonstrate how the numerical and symbolic tools can be applied to determine the asymptotic behavior of a nonlinear model. We find that this simple model inherits the asymptotic properties of the quadratic map. There are typically two fixed points, only one of which is an attracting fixed point.

When $\lambda=3.5$, for example, neither fixed point has the appropriate saddle point property. This does not mean that the model has no plausible perfect foresight solution. Then, the solution with the appropriate saddle point properties is a limit cycle of period three. 


\subsubsection{The Jullien Overlapping Generations Model}

$$
\begin{gathered}
H_{-1}=\left[\begin{array}{cc}
-\left(S_{w} W^{\prime}\right) & 0 \\
0 & 0
\end{array}\right] \\
H_{0}=\left[\begin{array}{cc}
1-S_{r} f^{\prime \prime} & 1 \\
-\left(f^{\prime \prime} m^{*}\right) & -r^{*}
\end{array}\right] \\
H_{1}=\left[\begin{array}{ll}
0 & 0 \\
0 & 1
\end{array}\right] \\
A=\left[\begin{array}{cc}
\frac{-\left(S_{w} W^{\prime}\right)+f^{\prime \prime} m^{*}}{-1+S_{r} f^{\prime \prime}} & \frac{r^{*}}{-1+S_{S} f^{\prime \prime}} \\
f^{\prime \prime} m^{*} & r^{*}
\end{array}\right]
\end{gathered}
$$

The eigenvalues are

$$
\frac{r^{*}+\frac{-\left(S_{w} W^{\prime}\right)+f^{\prime \prime} m^{*}}{-1+S_{r} f^{\prime \prime}} \pm \sqrt{\left(-r^{*}-\frac{-\left(S_{w} W^{\prime}\right)+f^{\prime \prime} m^{*}}{-1+S_{r} f^{\prime \prime}}\right)^{2}-4\left(-\frac{f^{\prime \prime} m^{*} r^{*}}{-1+S_{r} f^{\prime \prime}}+\frac{r^{*}\left(-\left(S_{w} W^{\prime}\right)+f^{\prime \prime} m^{*}\right)}{-1+S_{r} f^{\prime \prime}}\right)}}{2}
$$

One and only one of these must be greater than 1 for a unique solution converging to the steady state. If both are larger than one, then the trajectory of capital and money would converge to a limit cycle or behave chaotically.

Jullien [. jullien .] transforms this small dimensional system into a one dimensional system to prove that cycles and chaotic trajectories are possible. His global analysis exploits what is known about one dimensional maps to characterize the asymptotic properties of OLG models.

\subsubsection{A Certainty Equivalence Formulation of McCallum's Real Busi- ness Cycles Model}

McCallum [. barro business cycle .] describes a real business cycle model

$$
\begin{gathered}
c_{t}+k_{t}=z_{t} f\left(n_{t}, k_{t-1}\right)+(1-\delta) k_{t-1} \\
u_{1}\left(c_{t}, 1-n_{t}\right)-\lambda_{t}=0 \\
u_{2}\left(c_{t}, 1-n_{t}\right)=\lambda_{t} z_{t} f_{1}\left(n_{t}, k_{t-1}\right) \\
\lambda_{t}=\beta \lambda_{t+1}\left[z_{t+1} f_{2}\left(n_{t+1}, k_{t}\right)+1-\delta\right] \\
\left(z_{t}-z^{*}\right)=\rho\left(z_{t-1}-z^{*}\right)
\end{gathered}
$$

One can obtain an analytic expression for the state space transition matrix for solutions near a fixed point. The general expression is rather complicated, but particular functional forms would produce more easily interpretable expressions. 


\section{The Symbolic Computation Implementation of the Anderson Moore Saddle Point Analysis Routine Applied to the Overlapping Genera- tions Model}

This paper uses linear algebraic techniques to enhance the certainty equivalence solution techniques now in use. When non-linear models possess fixed points or limit cycles, a linear algebraic analysis of asymptotic properties can determine the local existence and uniqueness of convergent paths. In addition, linear algebraic analysis of the asymptotic behavior provides linear restrictions on the solution paths that are useful for computing non-linear solutions. The availability of symbolic algebra packages simplifies the once burdensome programming task associated with using non-linear models.

Augmenting the nonlinear certainty equivalence solution techniques with linear algebraic analysis of the asymptotic behavior helps to disentangle the several subtlety interacting sources of error which can arise in estimating nonlinear dynamic models and provides useful diagnostic tools and parameters for controlling the estimation routines.

The algorithm described above was originally designed for use in estimation of linear rational expectations models and for estimation of nonlinear certainty equivalence models. In that context, it is usually necessary and appropriate to use numerical linear algebra routines to compute the required matrices. In the present context it has proven worthwhile to use symbolic algebra programs to compute the matrices analytically.

In Benhabib and Laroque's model[. benhabib laroque .], individuals work in the first half of their lives and consume in the last half. Time $t$ workers choose $l_{t}, c_{t+1}, k_{t}$ to maximize a separable utility function given by

$$
U\left(c_{t+1}, l_{t}\right)=U_{1}\left(c_{t+1}\right)-U_{2}\left(l_{t}\right)
$$

subject to the budget constraints

$$
\begin{gathered}
k_{t}+M q_{t}=w_{t} l_{t} \\
c_{t+1}=r_{t+1} k_{t}+M q_{t+1}
\end{gathered}
$$

Benhabib and Laroque analyze the model with the firms production technology given by

$$
f\left(k_{t-1}, l_{t}\right)=A\left(\eta l_{t}^{-\rho}+(1-\eta) k_{t-1}^{-\rho}\right)^{-\frac{1}{\rho}}+(1-\delta) k_{t-1}
$$

Following Benhabib and Laroque, a certainty equivalence competitive equilibrium has

- $\left(l_{t}, c_{t+1}, k_{t}\right)$ is an optimal action for the consumer born at time $\mathrm{t}$ given the price system $\left(M, w_{t}, r_{t+1}, q_{t}, q_{t+1}\right)$. 
Figure 1:

- $\left(k_{t-1}, l_{t}\right)$ maximizes the profit of the firm under the technological constraint, given $\left(R_{t}, W_{t}\right)$.

- $F\left(k_{t-1}, l_{t}\right)=c_{t}+k_{t}$ for all $\mathrm{t}$.

The intratemporal variable interactions are displayed in figure 1

\subsection{The Basic Model}

The Benahabib and Laroque equation system 1.1 consists of:

Consumer budget constraint:

$$
k_{t}-F_{L}\left(k_{t-1}, l_{t}\right) l_{t}+M q_{t}=0
$$

Consumer arbitrage condition:

$$
-\left(F_{K}\left(k_{t}, l_{t+1}\right) q_{t}\right)+q_{t+1}=0
$$

Consumer maximization first order condition:

$$
F_{K}\left(k_{t}, l_{t+1}\right) F_{L}\left(k_{t-1}, l_{t}\right) U^{\prime}\left(F_{K}\left(k_{t}, l_{t+1}\right) F_{L}\left(k_{t-1}, l_{t}\right) l_{t}\right)-V^{\prime}\left(l_{t}\right)=0
$$

Lines 1 through 20 in appendix B show the mathematica commands which establish this equation system.

We need assumptions similar to those in Benhabib and Laroque to guarantee the existence of a fixed point.

Assumption $1 U$ is a strictly increasing concave function from $\Re_{+}$to $\Re$ and

$$
\lim _{C \rightarrow 0} U(C)=+\infty .
$$

$V$ is a strictly increasing convex function from $[0, \bar{L}]$ into $\Re$. It is smooth on $[0, \bar{L})$ and

$$
V^{\prime}(0)=0, \lim _{L \rightarrow \bar{L}} V^{\prime}(L)=+\infty
$$

Assumption $2 F$ is a strictly concave function from $\Re_{+}^{2}$ into $\Re_{+}$. It is homogeneous of degree 1 . It is smooth on the interior of $\Re_{+}^{2}$ and

$$
\begin{gathered}
\lim _{K \rightarrow \infty} F(K, L) / K \leq(1-\delta)<1 \\
\lim _{K \rightarrow 0} F_{K}(K, L)=\infty \forall L>0 \\
\lim _{L \rightarrow 0} F_{L}(K, L)=\infty \forall K>0
\end{gathered}
$$


Figure 2:

\subsection{Fixed Points}

\subsubsection{Existence and Uniqueness}

The fixed points for the system given by equations $8-9$ are given by the solution to: ${ }^{3}$

$$
\begin{gathered}
-V^{\prime}+U^{\prime} r^{*} w^{*}=0 \\
k^{*}+M q^{*}-l^{*} w^{*}=0 \\
q^{*}-q^{*} r^{*}=0
\end{gathered}
$$

where

$$
\begin{aligned}
w^{*} & =F_{L}\left[k^{*}, l^{*}\right] \\
r^{*} & =F_{K}\left[k^{*}, l^{*}\right]
\end{aligned}
$$

Thus there are two types of fixed point solutions.

- Monetary Solutions: $q^{*} \neq 0, r^{*}=1$

- Non-Monetary Solutions: $q^{*}=0$

I will illustrate the use of the symbolic processing techniques by analyzing the monetary solutions.

Monetary Solutions: $r^{\star}=1$ Assuming constant returns to scale we can find the marginal product of labor and the capital labor ratio corresponding to a marginal product of capital equal to one. In addition there exists a $\bar{K}$ such that $F_{K}(\bar{K}, \bar{L})=1$. As a result, we can express $\mu_{1}(k)=w^{*} U^{\prime}$ and $\mu_{2}(k)=V^{\prime}$ as functions of $k$. Figure 2 presents graphs illustrating the functions $\mu_{1}$ and $\mu_{2} . \mu_{1}(k)$ will be monotonically downward sloping with an asymptote for small values of $k$ and low consumption while $\mu_{2}(k)$ will be monotonically upward sloping with an asymptote at large values of $k$ and high labor.

The value of the capital stock where the functions $\mu_{1}$ and $\mu_{2}$ cross constitutes a fixed point. Uniqueness follows from the convexity of $\mathrm{V}$ and the concavity of $\mathrm{U}$.

\footnotetext{
${ }^{3}$ Lines 20 through 40 develop the equations presented here.
} 


\section{Asymptotic Stability of Monetary Fixed Points} $\quad U^{\prime}+U^{\prime \prime} l^{*} w^{*} \neq 0$ Assuming constant returns to scale so that $F_{K L} \rightarrow \frac{w^{*}}{\sigma y^{*}}$
and after making the simplifying substitutions $U^{\prime}+U^{\prime \prime} l^{*} w^{*} \rightarrow \phi$ and $V^{\prime \prime}-$ $U^{\prime \prime} w^{* 2} \rightarrow \tau$ we can write the minimal dimension OLG System transition matrix when $\phi \neq 0$ as:

$$
\begin{gathered}
{\left[\begin{array}{cccc}
0 & 1 & 0 & 0 \\
\frac{k^{*}+M q^{*}-\sigma y^{*}}{\sigma y^{*}} & \frac{l^{*} w^{*}}{k^{*}} & -\frac{\left(k^{*}+M q^{*}-\sigma y^{*}\right)\left(k^{*} \phi w^{*}+l^{*} \sigma \tau y^{*}\right)}{l^{*} \phi \sigma w^{*} y^{*}} & -M \\
-\frac{1}{w^{*}} & \frac{l^{*}}{k^{*}} & \frac{k^{*} \phi w^{*}+l^{*} \tau \tau y^{*}}{l^{*} \phi w^{*} 2} & 0 \\
-\frac{q^{*}}{\sigma y^{*}} & 0 & \frac{q^{*}\left(k^{*} \phi w^{*}+l^{*} \sigma \tau y^{*}\right)}{l^{*} \phi \sigma w^{*} y^{*}} & 1
\end{array}\right]} \\
{\left[\begin{array}{c}
k_{t-2} \\
k_{t-1} \\
l_{t-1} \\
q_{t-1}
\end{array}\right]}
\end{gathered}
$$

The characteristic polynomial is $\lambda$ times

$$
\begin{gathered}
1+\frac{l^{*} \tau}{\phi w^{*}} \\
\lambda\left(-1-\frac{k^{*}}{l^{*} w^{*}}-\frac{l^{*} \tau}{\phi w^{*}}-\frac{M l^{*} q^{*} \tau}{k^{*} \phi w^{*}}-\frac{l^{*} w^{*}}{k^{*}}-\frac{\sigma \tau y^{*}}{\phi w^{* 2}}\right) \\
\lambda^{2}\left(1+\frac{k^{*}}{l^{*} w^{*}}+\frac{l^{*} w^{*}}{k^{*}}+\frac{\sigma \tau y^{*}}{\phi w^{* 2}}\right) \\
-\lambda^{3}
\end{gathered}
$$

Since this equation is a cubic, we can obtain a closed form expression for each of the eigenvalues. Because such an expresssion would be rather long and complicated, subsection 4.2.2 will explore properties of the solutions by determining bifurcation values for the linearized system.

We can make further simplifications for monetary solutions. We can write the minimal dimension OLG System transition matrix as:

$$
\left[\begin{array}{cccc}
0 & 1 & 0 & 0 \\
-\frac{-1+\kappa+\sigma}{\sigma} & -1+\frac{1}{\kappa} & -\frac{(-1+\kappa+\sigma)(\kappa+\alpha \sigma) w^{*}}{(-1+\kappa) \sigma} & -M \\
-\frac{1}{w^{*}} & -\frac{-1+\kappa}{\kappa w^{*}} & -\frac{\kappa+\alpha \sigma}{-1+\kappa} & 0 \\
\frac{-1+2 \kappa}{M \sigma} & 0 & \frac{(-1+2 \kappa)(\kappa+\alpha \sigma) w^{*}}{M(-1+\kappa) \sigma} & 1
\end{array}\right]
$$

where $\kappa=\frac{k^{*}}{y^{*}}$ when the marginal product of capital is 1 .

The characteristic polynomial is $\lambda$ times $^{4}$

$$
1+\alpha
$$

\footnotetext{
${ }^{4}$ This is the same as Benhabib and Laroques' equation 3.1 if we set $\left\{r^{*} \rightarrow 1\right\}$ and $\left\{\tau \rightarrow-\frac{\alpha \phi w^{*}}{l^{*}}, \sigma \rightarrow \frac{l^{*} w^{*}}{\epsilon y^{*}}\right\}$
} 


\begin{tabular}{|c|l|}
\hline \multicolumn{2}{|c|}{ Bifurcations of Monetary Solutions $r^{*}=1$ Constant Returns to Scale } \\
\hline$\lambda=(1)$ & $-1+2 \kappa \neq 0 \wedge \alpha=0 \vee 2 \kappa=1$ \\
\hline$\lambda=(-1)$ & $1-\kappa+2 \kappa \sigma \neq 0 \wedge \alpha=\frac{-2}{1-\kappa+2 \kappa \sigma}$ \\
\hline$\lambda$ complex $|\lambda|=1$ & $\left(\frac{1}{2}<\kappa<1\right) \wedge\left(\alpha=\frac{1-2 \kappa}{-1+\kappa+\sigma}\right) \vee(\kappa=1 \wedge \alpha \sigma=-1)$ \\
\hline
\end{tabular}

Table 1:

$$
\begin{gathered}
\lambda\left(\alpha-\frac{1}{\kappa}-\frac{\alpha}{\kappa}-\frac{\kappa}{1-\kappa}-\frac{\alpha \sigma}{1-\kappa}\right) \\
\lambda^{2}\left(\frac{1}{\kappa}+\frac{\kappa}{1-\kappa}+\frac{\alpha \sigma}{1-\kappa}\right) \\
-\lambda^{3}
\end{gathered}
$$

\subsubsection{Bifurcations}

The asymptotic dynamics of the system near the fixed point are determined by the three parameters $\alpha, \kappa$, and $\sigma$. So long as there are exactly two roots outside the unit circle, there are unique trajectories which converge to the steady state. Combinations of parameters which correspond to unit roots are important because they trace out the border between regions where the fixed point has the appropriate stability properties and those regions where the stability properties are inappropriate.

We can use symbolic algebra programming to solve for combinations of the parameters which correspond to roots of unit modulus. Lines 140 to 179 solve for the combinations of parameters which characterize the bifurcation surface. Flip bifurcations occur when $\lambda=-1$. This occurs when $\alpha=\frac{-2}{1-\kappa+2 \kappa \sigma}$. Hopf bifurcations occurs for values of $\kappa>(1 / 2)$ when $\alpha=\frac{1-2 \kappa}{\kappa+\sigma-1}$. When $\kappa=(1 / 2)$ or $\alpha=0$ there is a root $\lambda=1$. Table 1 summarizes these results. The table generalizes the results Benhabib and Laroque obtained for a specific production to any constant returns to scale production function.

This paper demonstrates the feasibility and utility of applying symbolic numerical algebra programming for analyzing the asymptotic dynamics of non-linear economic models. The paper shows how one can adapt a numerical analysis program to produce analytic expressions for the asymptotic behavior of the nonlinear equation system. The symbolic algebra program provides a powerful tool for manipulating and solving the equation systems, verifying the solutions and recording the results. I have written the symbolic algebra programs in Mathematica and I have made them available via "anonymous ftp".

The paper also generalizes some of the results obtained by Benhabib and Laroque [. benhabib and laroque .]. The paper shows that, for any constant 
returns to scale production function, three parameters completely characterize the asymptotic behavior of the fixed points of the overlapping generations model. The paper provides formulae characterizing the combination of the three parameters where the asymptotic behavior of the model changes. 
A References 


\section{B Symbolic Program Code}

